\title{
Fixed point dual carrier modulation performance for wireless USB
}

Conference or Workshop Item

Published Version

Published conference article

Yang, R. and Sherratt, R. S. (2009) Fixed point dual carrier modulation performance for wireless USB. In: IEEE International Symposium on Consumer Electronics, June 2009, Kyoto, Japan, pp. 235-238. Available at http://centaur.reading.ac.uk/7478/

It is advisable to refer to the publisher's version if you intend to cite from the work. See Guidance on citing.

Published version at: http://ieeexplore.ieee.org/stamp/stamp.jsp?tp=\&arnumber $=5156913$

All outputs in CentAUR are protected by Intellectual Property Rights law, including copyright law. Copyright and IPR is retained by the creators or other copyright holders. Terms and conditions for use of this material are defined in the End User Agreement. 


\section{CentAUR}

Central Archive at the University of Reading

Reading's research outputs online 


\title{
Fixed Point Dual Carrier Modulation Performance for Wireless USB
}

\author{
Runfeng Yang and R. Simon Sherratt, Senior Member, IEEE
}

\begin{abstract}
Dual Carrier Modulation (DCM) is currently used as the higher data rate modulation scheme for Multiband Orthogonal Frequency Division Multiplexing (MB-OFDM) in the ECMA-368 defined Ultra-Wideband (UWB) radio platform. ECMA-368 has been chosen as the physical radio platform for many systems including Wireless USB (W-USB), Bluetooth 3.0 and Wireless HDMI; hence ECMA-368 is an important issue to consumer electronics and the user's experience of these products.

In this paper, Log Likelihood Ratio (LLR) demapping method is used for the DCM demaper implemented in fixed point model. Channel State Information (CSI) aided scheme coupled with the band hopping information is used as the further technique to improve the DCM demapping performance. The receiver performance for the fixed point DCM is simulated in realistic multi-path environments ${ }^{1}$.
\end{abstract}

\section{Index Terms - MB-OFDM, DCM, CSI. LLR}

\section{INTRODUCTION}

Ultra-Wideband (UWB) technology was historically employed in military radar systems. Recently UWB systems were proposed to standardize wide bandwidth wireless communication systems, particularly for Wireless Personal Area Networks (WPAN). The fundamental issue of UWB is that the transmitted signal can be spread over an extremely large bandwidth with a very low Power Spectral Density (PSD). In 2002, the USA Federal Communications Commission (FCC) agreed to allocate $7500 \mathrm{MHz}$ spectrum in 3.1-10.6 GHz band for unlicensed use for UWB devices [1] and limited the UWB Effective Isotropic Radiated Power (EIRP) to $-41.3 \mathrm{dBm} / \mathrm{MHz}$ [2].

In 2005 the WiMedia Alliance [3] working with the European Computer Manufacturers Association (ECMA) announced the establishment of the WiMedia MB-OFDM (Multiband Orthogonal Frequency Division Multiplexing) UWB radio platform as their global UWB standard, ECMA368. ECMA-368 was also chosen as physical layer (PHY) of high data rate wireless specifications for high-speed Wireless USB (W-USB) [4], Bluetooth 3.0 [5] and Wireless HighDefinition Media Interface (HDMI) [6]. Recently ECMA-368 has published a second updated version [7].

\footnotetext{
${ }^{1}$ This work was supported in part by The University of Reading Overseas Research Postgraduate Studentships.

Runfeng Yang is with the Signal Processing Laboratory (SPL), the University of Reading, RG6 6AY, UK (e-mail: r.yang@reading.ac.uk).

R. Simon Sherratt is with the Signal Processing Laboratory (SPL), the University of Reading, RG6 6AY, UK (e-mail: r.s.sherratt@ reading.ac.uk).
}

ECMA-368 specifies a MB-OFDM system occupying 14 bands. Each band with a bandwidth of $528 \mathrm{MHz}$ can support up to $480 \mathrm{Mb} / \mathrm{s}$. The first 12 bands are grouped into 4 band groups (BG1-BG4), and the last two bands are grouped into a fifth band group (BG5). A sixth band group (BG6) containing band 9,10 and 11 is also defined within the spectrum of BG3 and BG4, in agreement to use within worldwide spectrum regulations. The advantage of the grouping is that the transmitter and receiver can process a smaller bandwidth signal while taking advantages from frequency hopping.

The OFDM symbol is the basic quanta of MB-OFDM based UWB radio. Each OFDM symbol is constructed from the Inverse Fast Fourier Transform (IFFT) of a set of 128 complex valued carriers made from 100 data carriers, 12 pilot subcarriers, 6 NULL valued subcarriers and 10 guard subcarriers. The 10 guard subcarriers used for mitigating Inter Symbol Interference (ISI) are located on either edge of the OFDM symbol and they are the same value as the 5 outermost data subcarriers. In addition, the guard subcarriers can be used as another form of time and frequency diversity resulting in improved performance for the receiver [8]. Each OFDM symbol is appended with zero-padded suffix to aid multipath interference mitigation and settling times of the transmitter and receiver.

To operate the Physical layer (PHY) service interface to the Medium Access Control (MAC) service, a Physical Layer Convergence Protocol (PLCP) sublayer is defined to provide a method for converting a PSDU (PHY Service Data Unit) into a PPDU (PLCP Packet Data Unit) composed from three components (shown in Fig. 1): the PLCP preamble (containing the Packet/Frame Synchronization and the Channel Estimation sequence), the PLCP header, and the PSDU.

To transmit a Packet Service Data Unit (PSDU) that contains information bits, ECMA-368 has eight transmission modes by applying various levels of coding and diversity, which offers 53.3, 80, 106.7, 160, 200, 320, 400 or $480 \mathrm{Mb} / \mathrm{s}$ to the MAC layer. After the bit interleaver, the coded and interleaved binary data sequence is mapped onto a complex constellation. A Quadrature Phase Shift Keying (QPSK) constellation is used for data rates $200 \mathrm{Mb} / \mathrm{s}$ and lower. Dual Carrier Modulation (DCM) is used as a four-dimensional constellation for data rates $320 \mathrm{Mb} / \mathrm{s}$ and higher. DCM was introduced to the MB-OFDM proposal by Batra and Balakrishnan [9] as one of the enhancement changes to create the current WiMedia Alliance standard. 
PLCP Preamble

\begin{tabular}{|c|c|c|c|}
\hline Packet/Frame Sync & CE Sequence & PLCP Header & PSDU \\
\hline
\end{tabular}

Fig. 1. PPDU structure

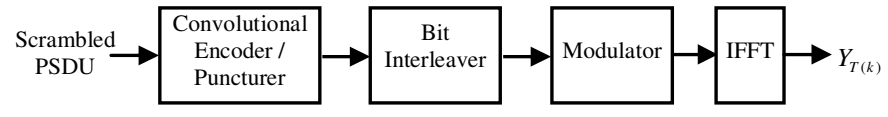

Fig. 2. Encoding process for the scrambled PSDU at Transmitter

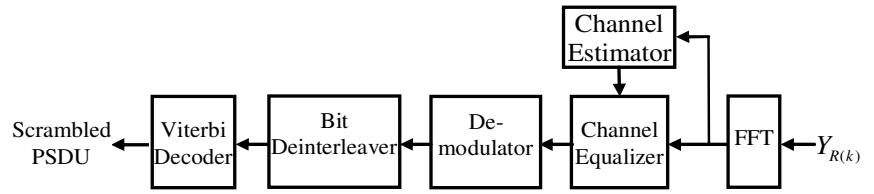

Fig. 3. Decoding process for the scrambled PSDU at Receiver

The resulting complex numbers are loaded onto the data subcarriers of the OFDM symbol implemented using an IFFT to create real or complex baseband signal. Fig. 2 and 3 depict the encoding and decoding process for the scrambled PSDU respectively. Chapter II introduces DCM mapping process. Chapter III discusses the DCM demaper using Log Likelihood Ratio (LLR) demapping method and Channel State Information (CSI) aided scheme for the further demapping technique. Chapter VI discusses the performance measurements and comparison while chapter VII presents the conclusions.

\section{DCM MAPPING}

\section{A. Frequency diversity}

Coded information on a single tone is unreliable if a channel has deep fade. However, the probability of experiencing a channel deep fade is extremely small if two tones with the same information are separated by a large bandwidth. Frequency diversity is used in the DCM by mapping the same coded information but different forms onto two different tones at different channel frequencies with a large bandwidth separation.

\section{B. DCM constellation mapping}

After bit interleaving, the 1200 interleaved and coded bits are divided into groups of 200 bits, and further grouped into 50 groups of 4 reordering bits. Each group of 4 bits is represented as $\left(\mathrm{b}_{\mathrm{g}(\mathrm{k})}, \mathrm{b}_{\mathrm{g}(\mathrm{k})+1}, \mathrm{~b}_{\mathrm{g}(\mathrm{k})+50}, \mathrm{~b}_{\mathrm{g}(\mathrm{k})+51}\right)$, where $\mathrm{k} \in$ $[0 . .49]$ and

$$
g(k)=\left\{\begin{array}{cc}
2 k & k \in[0 \ldots 24] \\
2 k+50 & k \in[25 \ldots 49]
\end{array}\right.
$$

These four bits are mapped to two QPSK symbols $\left(\mathrm{x}_{\mathrm{g}(\mathrm{k})}+\mathrm{j} \mathrm{x}_{\mathrm{g}(\mathrm{k})+50}\right),\left(\mathrm{x}_{\mathrm{g}(\mathrm{k})+1}+\mathrm{j} \mathrm{x}_{\mathrm{g}(\mathrm{k})+51}\right)$ as illustrated in (2).

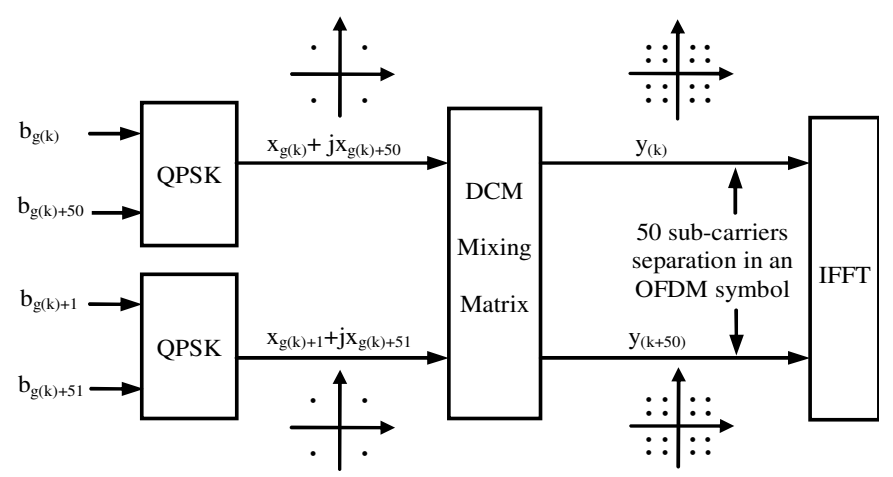

Fig. 4. DCM mapping process

$$
\left[\begin{array}{c}
x_{g(k)}+j x_{g(k)+50} \\
x_{g(k)+1}+j x_{g(k)+51}
\end{array}\right]=\left[\begin{array}{c}
\left(2 b_{g(k)}-1\right)+j\left(2 b_{g(k)+50}-1\right) \\
\left(2 b_{g(k)+1}-1\right)+j\left(2 b_{g(k)+51}-1\right)
\end{array}\right]
$$

Then the DCM mapper uses a DCM matrix $H$ as in (3) to execute mapping of the two QPSK symbols into two DCM symbols $\left(y_{\mathrm{T}(\mathrm{k})}, y_{\mathrm{T}(\mathrm{k}+50)}\right)$ as illustrated in (4). The resulting DCM symbols are formed into two 16-QAM like constellations [7].

$$
\begin{aligned}
& H=\left[\begin{array}{cc}
2 & 1 \\
1 & -2
\end{array}\right] \\
& {\left[\begin{array}{c}
y_{T(k)} \\
y_{T(k+50)}
\end{array}\right]=\frac{1}{\sqrt{10}}\left[\begin{array}{cc}
2 & 1 \\
1 & -2
\end{array}\right]\left[\begin{array}{c}
x_{g(k)}+j x_{g(k)+50} \\
x_{g(k)+1}+j x_{g(k)+51}
\end{array}\right]}
\end{aligned}
$$

where $1 / \sqrt{10}$ is a normalization factor for normalizing the average symbol power to be a constant unit.

The two resulting DCM symbols $\left(y_{\mathrm{T}(\mathrm{k})}, y_{\mathrm{T}(\mathrm{k}+50)}\right)$ are allocated into two individual OFDM data subcarriers with 50 sub-carriers separation to achieve frequency diversity. In total $100 \mathrm{DCM}$ symbols (complex numbers) are given to the $128 \mathrm{pt}$ IFFT block for building an OFDM symbol. Each OFDM subcarriers occupies a bandwidth of about $4 \mathrm{MHz}$. Therefore the bandwidth between the two individual OFDM data subcarriers related to the two complex numbers $\left(\mathrm{I}_{\mathrm{T}(\mathrm{k})}, \mathrm{Q}_{\mathrm{T}(\mathrm{k})}\right)$ and $\left(\mathrm{I}_{\mathrm{T}(\mathrm{k}+50)}, \mathrm{Q}_{\mathrm{T}(\mathrm{k}+50)}\right)$ is at least $200 \mathrm{MHz}$, which offers good frequency diversity gain against channel deep fading. Fig. 4 depicts the DCM mapping process.

\section{DCM DEMAPPING}

\section{A. Log likelihood ratio demapping}

The receiver converts each time-domain OFDM symbol into the frequency-domain via the Fast Fourier Transform (FFT). Then Channel Estimation and symbol Equalization follows. To demap the DCM symbols at the receiver, the received and equalized symbols previously transmitted on different sub-carriers can be demapped by using Log 
Likelihood Ratio (LLR). The DCM deampper uses two separate subcarriers concurrently to decode the symbol pair. If one symbol within one subcarrier is lost or degraded, it can be detected, even recovered by the DCM demapper. The proposed DCM soft demapping employs the DCM mixing matrix to combine the two equalized complex numbers into a sub-group of 4 soft bits. It is required to repeatedly execute demapping of the two received DCM sysmbols to output groups of 200 soft-bits. The soft bits from the DCM demapper are then inputted to the bit deinterleaver, the soft-bit Viterbi decoder and then descrambled to recover the PSDU. The generic format of LLR equation can be expressed in the following.

$$
L L R=\log (\exp (A)+\exp (B))-\log (\exp (X)+\exp (Y))
$$

In our case, a LLR is calculated from the received DCM symbols $y_{\mathrm{R}(\mathrm{k})}$ and $y_{\mathrm{R}(\mathrm{k}+50)}$. In addition, the LLR functions related to the two 16-QAM like constellations are independent. Hence the LLR for a group of 4 bits $\left(b_{g(k)}, b_{g(k)+1}\right.$, $\left.b_{g(k)+50}, b_{g(k)+51}\right)$ is formed from combining the two independent LLR, as in (6), (7), (8) and (9). $\sigma^{2}$ is noise variance associated with the channel.

$$
\begin{aligned}
& L L R\left(b_{g(k)}\right)=\log \left\{\sum_{b_{g(k)}=1} \exp \left[\frac{\left(I_{T(k)}-I_{R(k)}\right)^{2}}{-\sigma_{k}^{2}}\right]+\sum_{b_{s(k)}=1} \exp \left[\frac{\left(I_{T(k+50)}-I_{R(k+50)}\right)^{2}}{-\sigma_{k+50}^{2}}\right]\right\} \\
& -\log \left\{\sum_{b_{s(k)}=0} \exp \left[\frac{\left(I_{T(k)}-I_{R(k)}\right)^{2}}{-\sigma_{k}^{2}}\right]+\sum_{b_{s(k)}=0} \exp \left[\frac{\left(I_{T(k+50)}-I_{R(k+50)}\right)^{2}}{-\sigma_{k+50}^{2}}\right]\right\} \\
& L L R\left(b_{g(k)+1}\right)=\log \left\{\sum_{b_{s(k)+1}=1} \exp \left[\frac{\left(I_{T(k)}-I_{R(k)}\right)^{2}}{-\sigma_{k}^{2}}\right]+\sum_{b_{g(k)+1}=1} \exp \left[\frac{\left(I_{T(k+50)}-I_{R(k+50)}\right)^{2}}{-\sigma_{k+50}^{2}}\right]\right\}(7) \\
& -\log \left\{\sum_{b_{g(k)+1}=0} \exp \left[\frac{\left(I_{T(k)}-I_{R(k)}\right)^{2}}{-\sigma_{k}^{2}}\right]+\sum_{b_{g(k)+1}=0} \exp \left[\frac{\left(I_{T(k+50)}-I_{R(k+50)}\right)^{2}}{-\sigma_{k+50}^{2}}\right]\right\} \\
& L L R\left(b_{g(k)+50}\right)=\log \left\{\sum_{b_{g(k)+50}=1} \exp \left[\frac{\left(Q_{T(k)}-Q_{R(k)}\right)^{2}}{-\sigma_{k}^{2}}\right]+\sum_{b_{g(k)+50}=1} \exp \left[\frac{\left(Q_{T(k+50)}-Q_{R(k+50)}\right)^{2}}{-\sigma_{k+50}^{2}}\right]\right\}(8) \\
& -\log \left\{\sum_{b_{s(k)+50}=0} \exp \left[\frac{\left(Q_{T(k)}-Q_{R(k)}\right)^{2}}{-\sigma_{k}^{2}}\right]+\sum_{b_{s(k)+50}=0} \exp \left[\frac{\left(Q_{T(k+50)}-Q_{R(k+50)}\right)^{2}}{-\sigma_{k+50}^{2}}\right]\right\} \\
& L L R\left(b_{g(k)+51}\right)=\log \left\{\sum_{b_{g(k)+51}=1} \exp \left[\frac{\left(Q_{T(k)}-Q_{R(k)}\right)^{2}}{-\sigma_{k}^{2}}\right]+\sum_{b_{g(k)+51}=1} \exp \left[\frac{\left(Q_{T(k+50)}-Q_{R(k+50)}\right)^{2}}{-\sigma_{k+50}^{2}}\right]\right\}(9) \\
& -\log \left\{\sum_{b_{R(k)+51}=0} \exp \left[\frac{\left(Q_{T(k)}-Q_{R(k)}\right)^{2}}{-\sigma_{k}^{2}}\right]+\sum_{b_{R(k)+51}=0} \exp \left[\frac{\left(Q_{T(k+50)}-Q_{R(k+50)}\right)^{2}}{-\sigma_{k+50}^{2}}\right]\right\}
\end{aligned}
$$

For a Gaussian channel, the LLR can be approximated as two piecewise-linear functions which depend on the amplitude of I/Q signals [10], [11]. Furthermore, the maximum LLR value can be approximated to be soft magnitude with the associated bit completely depending on the amplitude of the I/Q signals. In our case, there are two bits associated with each of the two 16-QAM like constellations completely relying on their soft magnitude of the I/Q. Hence the LLR functions related to these two bits from each constellation are considered to be partially linear. Therefore some terms of these LLR functions are approximated by the proposed soft magnitude, as in (10), (11), (12) and (13).

$$
\begin{aligned}
& L L R\left(b_{g(k)}\right)=3 I_{R(k)}+\log \left\{\sum_{b_{s(k)}=1} \exp \left[\frac{\left(I_{T(k+50)}-I_{R(k+50)}\right)^{2}}{-\sigma_{k+50}^{2}}\right]\right\} \\
& -\log \left\{\sum_{b_{g(k)}=0} \exp \left[\frac{\left(I_{T(k+50)}-I_{R(k+50)}\right)^{2}}{-\sigma_{k+50}^{2}}\right]\right\} \\
& L L R\left(b_{g(k)+1}\right)=\log \left\{\sum_{b_{g(k)+1}=1} \exp \left[\frac{\left(I_{T(k)}-I_{R(k)}\right)^{2}}{-\sigma_{k}^{2}}\right]\right\} \\
& -\log \left\{\sum_{b_{s(k)+1}=0} \exp \left[\frac{\left(I_{T(k)}-I_{R(k)}\right)^{2}}{-\sigma_{k}^{2}}\right]\right\}-3 I_{R(k+50)} \\
& L L R\left(b_{g(k)+50}\right)=3 Q_{R(k)}+\log \left\{\sum_{b_{g(k)+50}=1} \exp \left[\frac{\left(Q_{T(k+50)}-Q_{R(k+50)}\right)^{2}}{-\sigma_{k+50}^{2}}\right]\right\} \\
& -\log \left\{\sum_{b_{R(k)+50}=0} \exp \left[\frac{\left(Q_{T(k+50)}-Q_{R(k+50)}\right)^{2}}{-\sigma_{k+50}^{2}}\right]\right\} \\
& L L R\left(b_{g(k)+51}\right)=\log \left\{\sum_{b_{s(k)+51}=1} \exp \left[\frac{\left(Q_{T(k)}-Q_{R(k)}\right)^{2}}{-\sigma_{k}^{2}}\right]\right\} \\
& -\log \left\{\sum_{b_{R(k)+51}=0} \exp \left[\frac{\left(Q_{T(k)}-Q_{R(k)}\right)^{2}}{-\sigma_{k}^{2}}\right]\right\}-3 Q_{R(k+50)}
\end{aligned}
$$

\section{B. Enhancement by exploiting Channel State Information}

In OFDM modulation, the OFDM subcarriers suffer from different noise powers, for example, echoes, deep fades, etc. Each OFDM subcarrier position has a dynamic estimation for the data reliability. This dynamic estimation in frequencydomain is defined as Channel State Information (CSI), which is used to enhance the channel decoder's error correction performance [12] [13]. Each data carrier has a potentially different CSI based on the power of the channel estimate at the corresponding frequency. The more reliable CSI is applied to the associated data subcarrier, the better decoding performance can be. The proposed CSI aided scheme coupled with the band hopping information maximizes the soft demapping performance [14] [15]. The resulting CSI is a scalar term indicative of the power of each frequency component of the sequences. The soft bits for the demodulator are scaled by the corresponding CSI, hence more reliable data for better decoding and achieve better system performance. As a result, each soft bit with incorporated CSI is derived as the following.

$$
\begin{aligned}
& \operatorname{Soft}\left(b_{g(k)}\right)=\operatorname{LLR}\left(b_{g(k)}\right) * \min \left\{\operatorname{CSI}_{k}, \operatorname{CSI}_{k+50}\right\} \\
& \operatorname{Soft}\left(b_{g_{(k)+1}}\right)=\operatorname{LLR}\left(b_{g_{(k)+1}}\right) * \min \left\{C S I_{k}, C S I_{k+50}\right\} \\
& \operatorname{Soft}\left(b_{g_{(k)+50}}\right)=\operatorname{LLR}\left(b_{g_{(k)+50}}\right) * \min \left\{C S I_{k}, C S I_{k+50}\right\} \\
& \operatorname{Soft}\left(b_{g(k)+51}\right)=\operatorname{LLR}\left(b_{g_{(k)+51}}\right) * \min \left\{C S I_{k}, C S I_{k+50}\right\}
\end{aligned}
$$




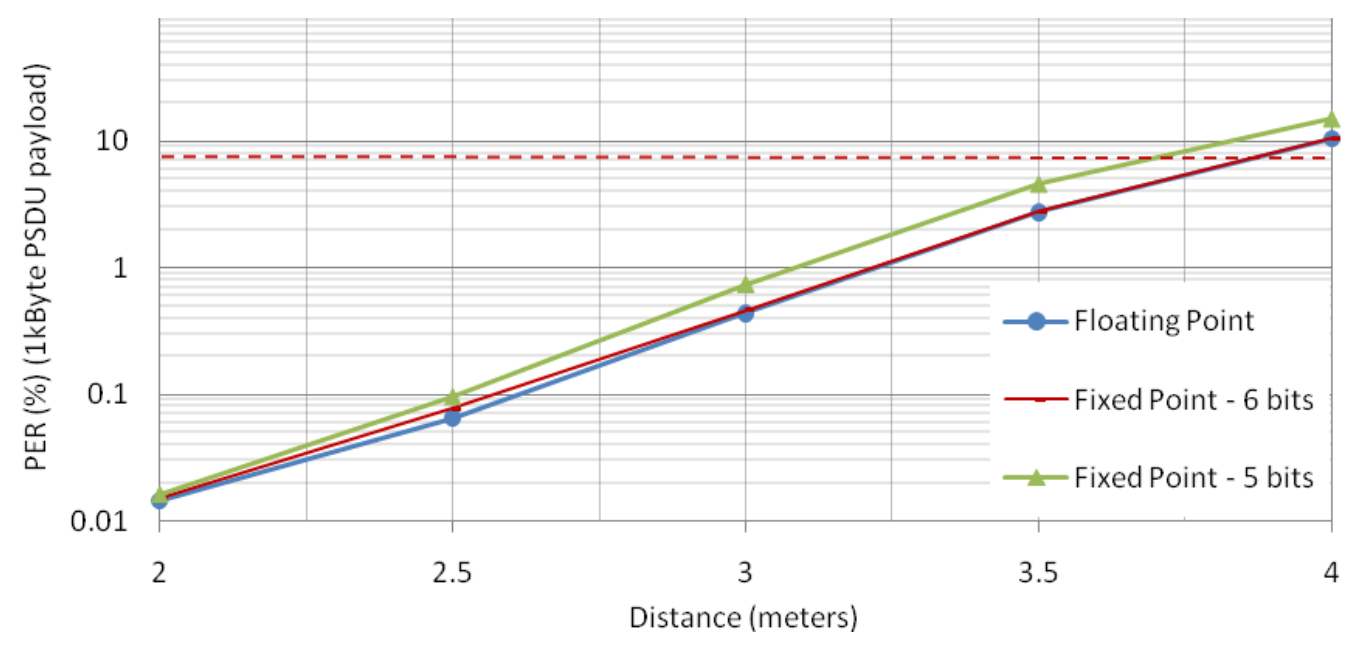

Fig. 5. System performance (in CM1) comparisons for fixed point and floating point DCM

\section{SYSTEM PERFORMANCE MEASUREMENTS}

The system is simulated in a realistic multipath channel environment of 100 channel realizations in Foerster's Channel Model 1 (CM1) [16] with conformance test requirement in ECMA-368. All simulations results are averaged over 2000 packets with 1024 octets per payload in the PSDU and 90thpercentile channel realization (the worst $10 \%$ channels are discarded). The link success probability is defined as system can be achieved with a Packet Error Rate (PER) less than $8 \%$ [17]. We maintain strict adherence to timing and use a hopping characteristic of Time Frequency Code $(\mathrm{TFC})=1$, and incorporate a Noise Figure (NF) of $6.6 \mathrm{~dB}$ and $2.5 \mathrm{~dB}$ implementation loss to the system model [7]. The simulation was performed with the use of guard pilot diversity resulting.

\section{V.CONCLUSION}

ECMA-368 offers a robust wireless solution and low cost wireless service in high speed WPAN. This paper presents DCM mapping and demapping processes for ECMA-368. Meanwhile, the fixed point DCM with 6 bits is found to maintain system performance with using the floating point DCM, which achieves a successful link of 3.9 meters at 480 $\mathrm{Mb} / \mathrm{s}$ in realistic multipath channel environment.

\section{REFERENCES}

[1] Federal Communications Commission, "New public safety applications and broadband internet access among uses envisiged by FCC authorization of ultra-wideband technology," press released $14^{\text {th }} \mathrm{Feb}$ 2002,http://www.fcc.gov/Bureaus/Engineering_Technology/News_Rele ases/2002/nret0203.html

[2] Federal Communications Commission, Revision of Part 15 of the Commissions Rules Regarding Ultra-Wideband Transmission Systems. First Report and Order, ET Docket 98-153, FCC 02-48; Adopted: February 14, 2002; Released: April 22, 2002. http://www. fcc.gov/Bureaus/Engineering_Technology/Orders/2002/fcc02048.pdf

[3] WiMedia Alliance http://www.wimedia.org/en/index.asp

[4] USB-IF. Certified Wireless USB. http://www.usb.org/developers/wusb/

[5] Bluetooth SIG. http://www.bluetooth.com/Bluetooth/Press/SIG

[6] Tzero Technologies, http://www.tzerotech.com/news/recent-news/
[7] ECMA-368, "High rate ultra wideband PHY and MAC standard," December 2007, http://www.ecma-international.org/publications/files/ ECMA-ST/ECMA-368.pdf

[8] R. S. Sherratt and R. Yang, "A dual QPSK soft-demapper for multiband OFDM exploiting time-domain spreading and guard interval diversity," IEEE Transactions on Consumer Electronics, vol. 53, no.1, pp. 46-49, February 2007

[9] A. Batra and J. Balakrishnan, "Improvements to the multi-band OFDM physical layer," 3rd IEEE Consumer Communications and Networking Conference, Volume 2, 8-10 Jan. 2006, pp. 701-705

[10] Steve Allpress, Carlo Luschi and Steve Felix, "Exact and Approximated Expressions of the Log-Likelihood Ratio for 16 QAM Signals," 38th Asilomar Conference on. Signals, Systems \& Computers, 7-10 Nov. 2004, Vol. 1, pp.794- 798

[11] F. Seguin, C. Lahuec, J.Lebert, M. Arzel and M. Jezequel, "Analogue 16 QAM Signals," IEE Electronics Letters, 2 Sept. 2004, Vol 40, Issue: 18, pp.1138- 1140

[12] W. Lee, H. Park, and Park J., "Viterbi decoding method using channel state information in COFDM system," IEEE Transactions on Consumer Electronics, vol. 45, no. 3, pp. 533-537, August 1999.

[13] W. Li, Z. Wang, Y. Yan and M. Tomisawa, "An efficient low-cost LS equalization in COFDM based UWB systems by utilizing channel-stateinformation (CSI)," Vehicular Technology Conference, 2005. VTC2005-Fall. 2005 IEEE 62nd, Sept, 2005, Vol. 4, pp. 67-71

[14] R. Yang and R. S. Sherratt, "An Improved DCM Soft-Demapper for the MB-OFDM UWB Platform Exploiting Channel-State-Information," IEEE/IET Signal Processing for Wireless Communications, London, 6-8 June 2007

[15] R. Yang and R. S. Sherratt, "Dual carrier modulation demapping methods and performances for Wireless USB," the 9th Annual Postgraduate Symposium (PGNET 2008), Liverpool,UK, June 2008

[16] J. Foerster, "Channel Modeling Sub-committee Report Final," IEEE P802.15 Working Group for Wireless Personal Area Networks (WPANs), IEEE P802.15-02/490-SG3a. 7th February 2003 http://grouper.ieee.org/groups/802/15/pub/2003/Mar03/02490r1P80215 SG3a-Channel-Modeling-Subcommittee-Report-Final.zip

[17] MBOA standard "MultiBand OFDM Physical Layer Proposal for IEEE 802.15.3a," September 2004, http://www.wimedia.org/imwp/idms/ popups/pop_download.asp?ContentID=6516

[all Accessed 30 ${ }^{\text {th }}$ March 2009] 